\title{
Experimental rat bladder urothelial cell carcinoma models
}

\author{
Harm C. Arentsen · Kees Hendricksen • \\ Egbert Oosterwijk $\cdot$ J. Alfred Witjes
}

Received: 12 January 2009 / Accepted: 26 January 2009 / Published online: 15 February 2009

(C) The Author(s) 2009. This article is published with open access at Springerlink.com

\begin{abstract}
Bladder cancer is a major public health problem. Currently available therapeutic options seem to be unable to prevent bladder cancer recurrence and progression. To enable preclinical testing of new intravesical therapeutic agents, a suitable bladder tumor model that resembles human disease is highly desirable. The aim of this topic paper was to discuss the problems associated with current in vivo animal bladder tumor models, focusing on the orthotopic syngeneic rat bladder tumor model. In the second part of the paper the development of a potential new orthotopic rat bladder tumor model is described.
\end{abstract}

Keywords Bladder cancer · Orthotopic $\cdot$ Rat $\cdot$ Model . Urothelial cell carcinoma

\section{Introduction}

Bladder cancer is one of the most common malignancies with approximately 357,000 new cases $(273,858$ males and 82,699 females) worldwide in 2002 [1]. More than $90 \%$ of the bladder cancers are urothelial cell carcinomas (UCC) [2] and the majority (75-85\%) presents initially as nonmuscle invasive bladder cancer (NMIBC) [3]. Of these patients, about $70 \%$ presents with papillary lesions that are non-invasive and limited to the mucosa (Ta), 20\% with lesions that invade the subepithelial connective tissue (T1) and $10 \%$ with carcinoma in situ (CIS). Transurethral resec-

H. C. Arentsen $(\varangle) \cdot$ K. Hendricksen · E. Oosterwijk · J. A. Witjes Department of Urology,

Radboud University Nijmegen Medical Centre, PO Box 9101, 6500 HB Nijmegen, The Netherlands e-mail: h.arentsen@uro.umcn.nl tion of the bladder tumor (TURBT), followed by adjuvant intravesical instillations with chemotherapy and/or immunotherapy are considered standard treatment for NMIBC. In general, the probabilities of recurrence and progression in NMIBC at 5 years range from 31 to $78 \%$ and from less than $1-45 \%$ depending on grade and stage, respectively [4]. These rates illustrate the modest success of currently available treatments and underline the need for improved adjuvant treatment.

Although in vitro models are useful for initial development and evaluation of therapeutic agents and modalities, adequate animal models are still essential in the preclinical development of new effective and safe therapies for many human malignancies. It allows the investigation of aspects that cannot be studied under clinical conditions such as pharmacokinetics and toxicity. For bladder cancer in particular, much effort has been devoted to the development of an appropriate tumor model for the evaluation of new chemotherapeutic or immunotherapeutic agents, drug regiments, or other anti-tumor modalities.

The ideal animal bladder tumor model that resembles human disease both histologically and in behavior should include the following characteristics [5]:

1. The tumor should grow intravesically (orthotopically), such that the tumor can be directly exposed to intravesical anti-tumor drugs in its natural environment.

2. The tumor should be of pure UCC origin, with different stages of disease progression (CIS, papillary and invasive diseases) and, as for the human disease, the majority of the tumors should be non-muscle invasive, but not progressive.

3. The animal host should be immunocompetent and reasonably large, so it can be treated by various anti-tumor modalities such as immunotherapy with bacillus 
Calmette-Guérin (BCG), chemotherapy, and whole bladder photodynamic therapy (PDT).

4. The tumor should be technically easy to develop within a reasonable time period, and highly reproducible with respect to its natural history.

In this topic paper, we discuss existing in vivo rat bladder tumor models with their specific shortcomings. In the second part of the paper, we describe the development of a potential new orthotopic rat bladder tumor model.

\section{Existing in vivo models}

Rats and mice are the most common species used for in vivo UCC models. Rodents have a lower urinary tract comparable to humans and neoplasms in the bladder are morphologically very alike [6]. Bladder tumors in rodents can be established subcutaneously (heterotopically) or in the bladder (orthotopically) either by transplantation of tumor cells or by chemical induction.

Although murine orthotopic models are available [e.g. 7, $8,9]$, these models are limited by the size of the animal, with a small urethral caliber, thin bladder wall and small bladder capacity as a consequence. Catheterization of mice to introduce tumor cells, intravesical therapeutics or for example an ultrathin cystoscope is more difficult than in rats. A rat bladder is approximately 10 times larger than the bladder of a mouse and the better developed muscular layer allows better histological assessment of depth of invasion and decreases the risk of perforation during bladder catheterization [10]. Therefore, in important aspects the rat model offers significant advantages over the murine model and in this topic paper only the rat model is further discussed.

\section{Subcutaneous models}

In rodent heterotopic UCC models, the tumor is usually located in the flank or hind leg of the animal. To establish a syngeneic subcutaneous model for immunological studies, rodent UCC tumor fragments or cells are inoculated through a small incision into the immunocompetent host of the same strain from which the tumor was originally derived $[11,12]$. For a xenograft model human UCC fragments or cells are used. Subcutaneous tumors can be evaluated non-invasively by palpation or with the help of imaging techniques. Treatment can be administered by local injection or systemically.

Subcutaneous bladder tumor models have been widely used because of the ease of assessing tumor growth kinetics and because the orthotopic model is technically more difficult (see below) $[13,14]$. However, the microenvironment at the implantation site of the host organ can influence the natural history of tumor growth and the efficacy of anti-proliferative agents. In one study, for example [15], human colon carcinoma cells were implanted into different anatomical locations (subcutaneous and cecum) of nude mice. Tumor bearing mice were treated with doxorubicin and subsequently evaluated for responses. The heterotopic subcutaneous tumors showed an $80 \%$ inhibition of growth, compared with $40 \%$ inhibition of the orthotopic intracecal tumors. The environment of the orthotopic model resembles that of naturally occurring tumors, so experimental results in this model would be expected to have more relevance than results in the subcutaneous model. In addition, heterotopic grafts are unsuited to evaluate intravesical therapies and orthotopic models are thus to be preferred.

Orthotopic models: tumor development

To test potential new drugs against NMIBC in a preclinical setting, a clinically relevant rodent orthotopic bladder tumor model is highly desirable. Currently, there are three different kinds of orthotopic rat bladder tumor models: chemically induced bladder cancer models [16], the xenograft model (transplantation of human UCC into immunodeficient nude rats) [10] and the syngeneic tumor model (transplantation of carcinogen-induced bladder cancer in syngeneic immunocompetent rats) [5].

Given the importance of chemicals in the carcinogenesis of bladder cancer, organo-specific bladder carcinogens were discovered for rodents. These chemicals provided readily available reproducible models necessary for detailed studies of the pathogenesis of bladder cancer [17]. Three chemicals have been reported as being particularly effective in causing bladder tumors under the appropriate conditions: $\quad$ FANFT ( $N$-[4-(5-nitro-2-furyl)-2-thiazolyl]formamide), OH-BBN ( $N$-butyl- $N$-(4-hydroxybutyl)nitrosamine) and MNU ( $N$-methyl- $N$-nitrosurea) [18, 19]. However, the use of chemicals to induce tumors in the rat bladder is time-consuming (several months) and there is a high amount of squamous differentiation. Furthermore, reproducibility is hampered by the variation in time to tumor development. Therefore, these models are less practical in therapeutic efficacy studies of novel anti-proliferative compounds.

Bladder tumor transplantation is a much faster approach to create a therapeutic in vivo orthotopic UCC model. Orthotopic xenografts in immunodeficient nude rats are useful in order to better approximate human tumor cell behavior in situ, but are unsuited to test intravesical immunotherapy with for example BCG and are not frequently used.

Xiao et al. [5]described a syngeneic orthotopic rat model resembling human UCC, with reproducible tumor growth. 
The rat bladder UCC cell line AY-27 was established as a primary bladder tumor in Fischer F344 rats by feeding FANFT. This rat bladder UCC cell line was transplanted orthotopically into female Fischer F344 rats. AY-27 UCC cells were grown in monolayer cell culture and instilled intravesically as single cell suspensions into bladders that had been preconditioned with mild acid washing. In 82 animals sacrificed at 16 days, 80/82 (97\%) developed UCC, and in $52 / 80(65 \%)$ the tumor was non-muscle invasive (CIS, T1, but no Ta). Histological examination of the tumor specimens confirmed the presence of grade II-III UCC. Immunocytochemistry confirmed that the tumor model maintained the features of UCC.

A potential demerit in this frequently used model is the timing of intravesical therapy after tumor cell inoculation: in some rats tumors had not formed yet, while other rats already developed muscle invasive tumors. Therefore, Hendricksen et al. [20] assessed the growth of UCC in this model over time, aiming to have a maximum number of rats with NMIBC at a particular time and thus optimize the future starting point of experimental intravesical treatment. It was shown that in this model tumors were formed at 3-5 days and remained non-muscle invasive up to 5 days. From 6 days, tumors progressed to muscle-invasive disease in $40 \%$ of the rats. The observed increase in tumor aggressiveness might be explained by more passages in vitro.

Another disadvantage of transplantable models is that tumors prove to be invasive carcinomas ( $\geq \mathrm{T} 1)$ from the start, without any Ta tumors (which is the most common stage in human NMIBC). This is related to the bladder preconditioning with urothelial abrasion rather than to the aggressiveness of the AY-27 cell line. It is quite possible that the basal membrane is disrupted, which causes rapid invasion of tumor cells into the underlying layers of the bladder. However, tumor cells instilled into normal (nonconditioned) bladders did not result in tumor establishment [5]. Different methods of tumor establishment in orthotopic models have been described, ranging from intravesical instillation of tumor cell suspension after chemical urothelial denudation with $\mathrm{MNU}$ or the combination of $\mathrm{HCl}$ and $\mathrm{KOH}$ leading to multifocal lesions, to mechanical urothelial lesioning such as cauterization [21]. Intraparietal injection of tumor cells resulted in intramuscular tumors only [22, 23]. Horiguchi et al. [24], however, reported also promising success rates without bladder preconditioning. Thus, the model could possibly be improved by controlling the urothelial abrasion: the lesion should be only superficial, affecting only one or two of the three cell layers of the rat bladder urothelium. On the other hand, if one assumes that tumor cell implantation on the urothelium injured during TURBT is a major cause of bladder cancer recurrence in humans, bladder preconditioning would resemble the clinical situation in case the model is used to test immediate postoperative intravesical treatments.

Orthotopic models: tumor evaluation

For proper determination of treatment response, confirmation of successful orthotopic tumor implantation before starting treatment is desirable. Otherwise one has to rely on former experiments and conjecture stage and tumor take. Since there is no accurate documentation of the presence or extent of tumor before therapy in this case, the validity of any conclusion on the efficacy of the experimental agents is difficult and large experimental groups are needed to reach enough power. If the autopsy specimen after treatment contained no tumor one could not conclude whether complete tumor regression was caused by therapy or whether tumor implantation had failed. Therefore, a placebo group should always be added in treatment efficacy experiments in case successful tumor implantation before starting treatment could not be assessed. In any case, there is the disadvantage of consuming more animals, either due to larger treatment groups or to adding a placebo group.

Precise quantification of bladder tumor lesions is also needed to assess responses to intravesical instillation of therapeutic agents. Accurate non-invasive in vivo assessment of established tumors in the orthotopic model is difficult, particularly for early stage non-muscle invasive tumors. Magnetic resonance imaging (MRI) has been reported for this purpose $[25,26]$. However, until now it could not offer an accurate diagnosis of small early lesions ( $<1 \mathrm{~mm}$ in diameter) due to its spatial resolution. Furthermore, it is relatively complicated and has high costs. Intravesical ultrasonography was also reported [27], and provided a positive-predictive ratio of tumor stage up to $85 \%$, however, without information on tumor location and appearance. Bioluminescence imaging has the potential to become a valuable tool for the early detection of tumor growth, although bioluminescence was only first detectable on day 4 after tumor cell implantation in recently published studies [28, 29]. Ultrathin cystoscopy [30] is a reliable method in diagnosing tumor growth in an orthotopic rat bladder tumor model, with a specificity and sensitivity of $>90 \%$ [20]. Unfortunately, in our own experience, cystoscopy is suboptimal in the follow-up of tumors after intravesical treatment in this model. Most probably the rather short interval between intravesical instillation and cystoscopy, necessary because of the fast tumor development, hampers reliable cystoscopy. This lack of a reliable method to follow-up tumor development makes it difficult to study treatment outcomes without sacrificing the animals, because the histological examination of the bladder appears to remain the golden standard for the determination of tumor growth. 


\section{Potential new orthotopic ACI rat bladder tumor (RBT323) model}

In the search for the ideal animal bladder cancer model, we are currently developing a new orthotopic ACI rat bladder tumor (RBT323) model. The RBT323 tumor is a non-carcinogen-induced tumor and arose spontaneously in an ACI rat. In the past, this RBT323 tumor was used as a subcutaneous rat bladder tumor model in our laboratory [31, 32]. Histologically, the RBT323 tumor is a grade 2-3 pure urothelial cell tumor with no squamous cells and the RBT323 cell line closely resembles human non-muscle invasive UCC both phenotypically and cytogenetically [11].

The rather short treatment window of the frequently used syngeneic orthotopic AY-27/Fischer F344 rat bladder tumor model hampers its use in the evaluation of new treatments for NMIBC [5]. Moreover, it was recently shown that carcinogen-induced rodent bladder tumors have gene expression profiles more similar to those of human invasive than non-invasive tumors and thus would be a better experimental model for the former [33]. For these reasons, we anticipated an orthotopic RBT323 tumor in the ACI rat to be an appropriate syngeneic model to study the anti-tumor effects of possible new treatments.

To accomplish this, a RBT323 rat bladder UCC cell line was established and transplanted orthotopically into syngeneic female ACI/SeqHSD rats (Harlan Spraque Inc., Indianapolis, Ind.). RBT323 UCC cells were grown in monolayer cell culture and instilled into preconditioned (mild acid washing) rat bladders as single cell suspensions. Tumor growth was assessed by ultrathin cystoscopy $(0,5 \mathrm{~mm}$ diameter) and 24 days after instillation of tumor cells all rats were sacrificed and subjected to necropsy. Cystectomy was performed for histopathological examination. Overall in three out of the four rats tumor was established. All tumors were non-muscle invasive (T1a-T1c) except one T2a tumor (2002 TNM staging system). No metastases were found. Unfortunately, all tumors were covered with normal urothelium (Fig. 1). Still, initial results of this new orthotopic rat bladder tumor model are promising, because after 24 days most of the tumors seem to be non-muscle invasive with a reasonably long time window for the administration of study drugs, as a consequence. Further studies are now being performed to look for the reproducibility and longitudinal behavior of the model. Additionally, a study is performed without bladder preconditioning, but with optimized other transplantation conditions, trying to establish Ta tumors.

\section{Conclusion}

To enable efficacy testing of new potential chemotherapeutic or immunotherapeutic agents, an orthotopic syngeneic

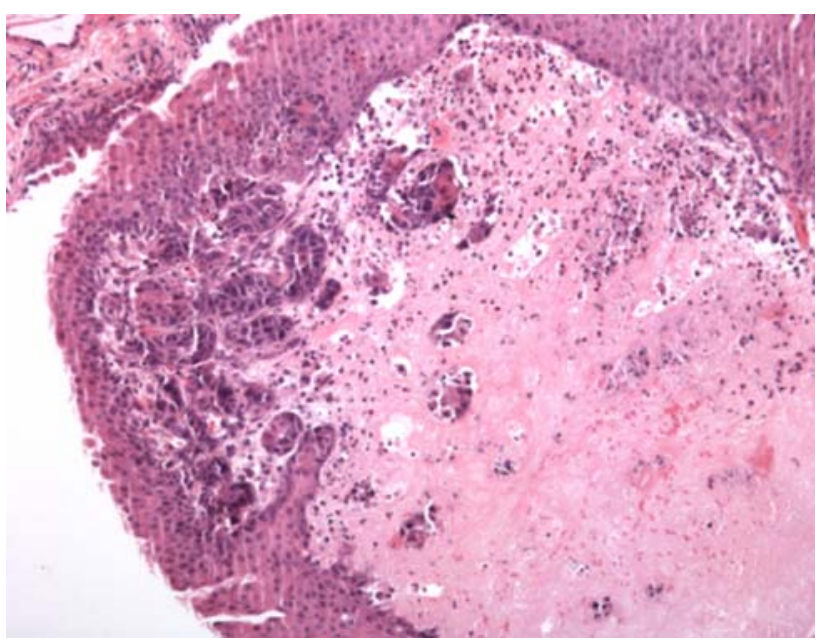

Fig. 1 RBT323 rat UCC T1a tumor, with overlying normal urothelium

non-muscle invasive UCC rat bladder tumor model would be ideal. However, especially tumor cell implantation methods and diagnostic procedures for the early detection of tumor growth, and follow-up monitoring still need to be refined.

Conflict of interest statement No potential conflict of interest relevant to this article was reported.

Open Access This article is distributed under the terms of the Creative Commons Attribution Noncommercial License which permits any noncommercial use, distribution, and reproduction in any medium, provided the original author(s) and source are credited.

\section{References}

1. Curado MP, Edwards B, Shin HR et al (2007) Cancer incidence in five continents, vol IX. IARC Scientific Publications No.160. IARC, Lyon

2. Fleshner NE, Herr HW, Stewart AK, Murphy GP, Mettlin C, Menck HR (1996) The national cancer data base report on bladder carcinoma. Cancer 78:1505-1513. doi:10.1002/(SICI)10970142(19961001)78:7<1505::AID-CNCR19>3.0.CO;2-3

3. Babjuk M, Oosterlinck W, Sylvester R, Kaasinen E, Böhle A, Palou J (2008) Guidelines on TaT1 (non-muscle invasive) bladder cancer. Eur Urol 54:303-314. doi:10.1016/j.eururo.2008.04.051

4. Sylvester RJ, van der Meijden AP, Oosterlinck W et al (2006) Predicting recurrence and progression in individual patients with stage Ta T1 bladder cancer using EORTC risk tables: a combined analysis of 2596 patients from seven EORTC trials. Eur Urol 49:466-477. doi:10.1016/j.eururo.2005.12.031

5. Xiao Z, McCallum TJ, Brown KM et al (1999) Characterization of a novel transplantable orthotopic rat bladder transitional cell tumour model. Br J Cancer 81:638-646. doi:10.1038/sj.bjc.6690741

6. Oyasu R (1995) Epithelial tumours of the lower urinary tract in humans and rodents. Food Chem Toxicol 33:747-755. doi:10.1016/ 0278-6915(95)00042-Z

7. Günther JH, Jurczok A, Wulf T et al (1999) Optimizing syngeneic orthotopic murine bladder cancer (MB49). Cancer Res 59:28342837 
8. Watanabe T, Shinohara N, Sazawa A et al (2000) An improved intravesical model using human bladder cancer cell lines to optimize gene and other therapies. Cancer Gene Ther 7:1575-1580. doi:10.1038/sj.cgt.7700261

9. Chong L, Ruping Y, Jiancheng B et al (2006) Characterization of a novel transplantable orthotopic murine xenograft model of a human bladder transitional cell tumor (BIU-87). Cancer Biol Ther 5:394-398

10. Oshinsky GS, Chen Y, Jarrett T, Anderson AE, Weiss GH (1995) A model of bladder tumor xenografts in the nude rat. J Urol 154:1925-1929. doi:10.1016/S0022-5347(01)66827-7

11. Van Moorselaar RJA, Ichikawa T, Schaafsma HE et al (1993) The rat bladder tumor model system RBT resembles phenotypically and cytogenetically human superficial transitional cell carcinoma. Urol Res 21:413-421. doi:10.1007/BF00300078

12. Moltedo B, Faunes F, Haussmann D et al (2006) Immunotherapeutic effect of Concholepas hemocyanin in the murine bladder cancer model: evidence for conserved antitumor properties among hemocyanins. J Urol 176:2690-2695. doi:10.1016/j.juro.2006. 07.136

13. Talmadge JE, Singh RK, Fidler IJ, Raz A (2007) Murine models to evaluate novel and conventional therapeutic strategies for cancer. Am J Pathol 170:793-804. doi:10.2353/ajpath.2007.060929

14. Bibby MC (2004) Orthotopic models of cancer for preclinical drug evaluation: advantages and disadvantages. Eur J Cancer 40:852857. doi:10.1016/j.ejca.2003.11.021

15. Wilmanns C, Fan D, O'Brian C et al (1993) Modulation of doxorubicin sensitivity and level of P-glycoprotein expression in human colon carcinoma cells by ectopic and orthotopic environments in nude mice. Int J Oncol 3:413-422

16. Grubbs CJ, Lubet RA, Koki AT et al (2000) Celecoxib inhibits NButyl-N-(4-hydroxybutyl)-nitrosamine-induced urinary bladder cancers in male B6D2F1 mice and female Fischer-344 rats. Cancer Res 60:5599-5602

17. Cohen SM (1998) Cell proliferation and carcinogenesis. Drug Metab Rev 30:339-357. doi:10.3109/03602539808996317

18. Schalken JA, Van Moorselaar RJA, Bringuier PP, Debruyne FMJ (1992) Critical review of the models to study the biologic progression of bladder cancer. Semin Surg Oncol 8:274-278. doi:10.1002/ ssu.2980080505

19. Oliveira PA, Colaço A, De la Cruz LF, Lopes C (2006) Experimental bladder carcinogenesis-rodent models. Exp Oncol 28:2-11

20. Hendricksen K, Molkenboer-Kuenen J, Oosterwijk E, Hulsbergen-van de Kaa CA, Witjes JA (2008) Evaluation of an orthotopic rat bladder urothelial cell carcinoma model by cystoscopy. BJU Int 101:889-893. doi:10.1111/j.1464-410X.2007.07407.x

21. Bisson J, Parache RM, Droulle P, Notter D, Vigneron C, Guillemin F (2002) A new method of implanting orthotopic rat bladder tumor for experimental therapies. Int J Cancer 102:280 285. doi:10.1002/ijc. 10595

22. Iinuma S, Bachor R, Flotte T, Hasan T (1995) Biodistribution and phototoxicity of 5-aminolevulinic acid-induced PpIX in an orthotopic rat bladder tumor model. J Urol 153:802-806. doi:10.1016/ S0022-5347(01)67726-7

23. Bindels EMJ, Vermey M, De Both NJ, Van der Kwast TH (2001) Influence of the microenvironment on invasiveness of human bladder carcinoma cell lines. Virchows Arch 439:552-559. doi:10.1007/s004280100419

24. Horiguchi Y, Kikuchi E, Ozu C et al (2008) Establishment of orthotopic mouse superficial bladder tumor model for studies on intravesical treatments. Hum Cell 21:57-63. doi:10.1111/j.17490774.2008.00055.x

25. Chin J, Kadhim S, Garcia B, Kim YS, Karlik S (1991) Magnetic resonance imaging for detecting and treatment monitoring of orthotopic murine bladder tumor implants. J Urol 145:1297-1301

26. Kikuchi E, Xu S, Ohori M et al (2003) Detection and quantitative analysis of early stage orthotopic murine bladder tumor using in vivo magnetic resonance imaging. J Urol 170:1375-1378. doi:10.1097/01.ju.0000075504.13456.41

27. Satoh H, Morimoto Y, Arai T et al (2007) Intravesical ultrasonography for tumor staging in an orthotopically implanted rat model of bladder cancer. J Urol 177:1169-1173. doi:10.1016/j.juro. 2006.10.038

28. Hadaschik BA, Black PC, Sea JC et al (2007) A validated mouse model for orthotopic bladder cancer using transurethral tumor inoculation and bioluminescence imaging. BJU Int 100:13771384. doi:10.1111/j.1464-410X.2007.07165.x

29. Jurczok A, Fornara P, Söling A (2007) Bioluminescence imaging to monitor bladder cancer cell adhesion in vivo: a new approach to optimize a syngeneic orthotopic, murine bladder cancer model. BJU Int 101:120-124

30. Asanuma H, Arai T, Seguchi K et al (2003) Successful diagnosis of orthotopic rat superficial bladder tumor model by ultrathin cystoscopy. J Urol 169:718-720. doi:10.1016/S0022-5347(05) 64000-1

31. Van Moorselaar RJA, Hendriks BT, Borm G, Van der Meide PH, Debruyne FMJ, Schalken JA (1992) Inhibition of rat bladder tumor (RBT323) growth by tumor necrosis factor alpha and interferon-gamma in vivo. J Urol 148:458-462

32. Cornel EB, Van Moorselaar RJA, Van Stratum P, Debruyne FMJ, Schalken JA (1993) Antitumor effects of Bacillus Calmette-Guerin in a syngeneic rat bladder tumor model. J Urol 149:179-182

33. Williams PD, Lee JK, Theodorescu D (2008) Molecular credentialing of rodent bladder carcinogenesis models. Neoplasia $10: 838-846$ 